\title{
Lifshitz formula for the Casimir force and the Gelfand-Yaglom theorem
}

\author{
C. Ccapa Ttira ${ }^{a}$, C. D. Fosco ${ }^{b, c}$, and F. D. Mazzitelli ${ }^{c, d}$ \\ ${ }^{a}$ Instituto de Fisica - UFRJ \\ CP 68528, 21941-972 Rio de Janeiro, RJ, Brazil \\ ${ }^{b}$ Instituto Balseiro \\ Universidad Nacional de Cuyo \\ R8402AGP Bariloche, Argentina. \\ ${ }^{c}$ Centro Atómico Bariloche \\ Comisión Nacional de Energía Atómica \\ R8402AGP Bariloche, Argentina \\ ${ }^{d}$ Departamento de Física \\ Facultad de Ciencias Exactas y Naturales - Universidad de Buenos Aires \\ Ciudad Universitaria, Pabellón 1, 1428 Buenos Aires, Argentina.
}

\begin{abstract}
We provide a Quantum Field Theory derivation of Lifshitz formula for the Casimir force due to a fluctuating real scalar field in $d+1$ dimensions. The field is coupled to two imperfect, thick, plane mirrors, which are modeled by background potentials localized on their positions. The derivation proceeds from the calculation of the vacuum energy in the Euclidean version of the system, reducing the problem to the evaluation of a functional determinant. The latter is written, via Gelfand-Yaglom's formula, in terms of functions depending on the structure of the potential describing each mirror; those functions encode the properties which are relevant to the Casimir force and are the reflection coefficients evaluated at imaginary frequencies.
\end{abstract}

\section{Introduction}

Just a few years after Casimir's calculation of the attractive force between ideal metallic plates [1, Lifshitz derived his celebrated formula for the inter- 
action of bodies with arbitrary, frequency-dependent dielectric functions [2]. In the original calculation, Lifshitz considered two disjoint media-filled halfspaces with plane and parallel boundaries. The calculation was performed at finite temperature and the final result for the interaction force was written in terms of the dielectric functions that describe, macroscopically, the electromagnetic properties of each media.

The impressive refinement achieved in precision experiments measuring the Casimir force stimulated a large amount of further theoretical calculations. Indeed, to explain recent experimental results it has become increasingly important to use models that describe the mirrors in a more realistic way. For example, the dependence of the Casimir interaction on the geometry, temperature, and macroscopic electromagnetic properties of the interacting bodies is a subject of growing interest [3].

As an outcome of the sustained research effort on these topics, Lifshitz formula has been generalized in several directions. In this work, we shall focus on its generalization to the case of stratified media, i.e. a set of plane-parallel layers of different materials. The interaction between the different slabs can be computed and expressed in terms of the electromagnetic properties of the layers or, more specifically, in terms of their reflection coefficients, which generally depend on the field's frequency and momentum.

Lifshitz formula, and some of its generalizations, have been derived using various different theoretical approaches. In the original setting [2], Lifshitz introduced a random field in the Maxwell equations, in order to describe the fluctuating sources in the materials. The interaction between bodies was computed by evaluating the appropriate component of the stress tensor, after assuming a local correlation for the random field. Besides, the existence of different media was taken into account by imposing the corresponding boundary conditions for the Green's functions of the electromagnetic field. This formula has been recently rederived [4] using a canonical quantization approach for macroscopic QED, in which the starting point is the action of the electromagnetic field, coupled to a set of reservoir oscillators. An alternative approach [5], is to quantize the electromagnetic field in the presence of the plane-parallel layers, computing the zero-point energy by following Casimir's original line of thought. Then the boundary conditions on the modes of the electromagnetic field determine the allowed eigenfrequencies, and the summation of the zero-point energy of each mode is performed using the argument theorem. Note that, in this derivation, the validity of Lifshitz formula in lossy media is not apparent.

Lifshitz formula has also been derived in a quite different context, namely, considering the mirrors, and the spaces between them, as a quantum optical network [6]. Within this formalism it becomes possible to compute the force 
between lossy mirrors, and the final answer can be expressed just in terms of the frequency-dependent reflection coefficients of each mirror. Remarkably, these coefficients are the only properties of the media that become relevant to the computation of the vacuum force, in spite of the fact that the same coefficient may be obtained from different kinds of mirrors.

The Casimir force for absorbing media has also been considered in Ref. [7], where Lifshitz formula has been derived using the theory of quantum open systems.

Another route to the computation of the Casimir force is to consider a vacuum field in the presence of background potentials localized on the mirrors [8, 9]. These models can be justified from a microscopic point of view taking into account the interaction of the internal degrees of freedom of the mirrors with the vacuum field [9, 10]. Using a functional approach, the integration of the internal degrees of freedom of the mirror produces an effective action for the vacuum field. That effective action contains a potential that is different from zero just at the positions of the slabs. These models may be used to reproduce different boundary conditions for the fields which approach perfect ones only under certain specific limits. Although localized on each mirror, the potentials are in general nonlocal in time as well as in the coordinates parallel to the mirror. In other words, the boundary conditions for the Fourier modes of the field may depend on frequency or momentum.

In this kind of approach, the zero-point energy is given by the functional determinant of an operator containing the potentials that describe the media, and the formal general expression seems to be strongly dependent on the specific shape of the potentials involved. This is however, a puzzling circumstance. As already mentioned, Lifshitz formula is a quite general expression for the Casimir force between two plane and parallel mirrors, and the force depends on the potentials only through their reflection coefficients. In this paper we show, by providing a field theoretic derivation of the Lifshitz formula, that that is indeed the case.

The derivation relies upon the use of Gelfand-Yaglom formula for functional determinants [11] (for a modern review, see [12]). As a by-product, we shall find an expression which also holds true in situations where an uncritical application of Lifschitz formula may be problematic, namely, potentials leading to bound states.

This paper is organized as follows: in Section 2 we define the kind of system we consider and derive an expression for the Casimir force. Then in Section 3 we discuss some consequences of the general result, considering some particular examples. Our conclusions are presented in Section 4. 


\section{Derivation of the Casimir force}

Throughout this paper, we shall consider the case of a single, massive, real scalar vacuum field, $\varphi$, in $d+1$ spacetime dimensions, equipped with an Euclidean action $S(\varphi)$, which has the structure:

$$
\mathcal{S}(\varphi)=\mathcal{S}_{0}(\varphi)+\mathcal{S}_{I}(\varphi)
$$

where $S_{0}$ is the free field action

$$
\mathcal{S}_{0}(\varphi)=\frac{1}{2} \int d^{d+1} x\left[(\partial \varphi)^{2}+m^{2} \varphi^{2}\right]
$$

while $S_{I}$ describes the interaction between the field and two mirrors. Those mirrors are assumed to be plane and parallel, with their normals pointing along the $x_{d}$ direction (the remaining directions will be denoted by $x_{0}$ and $\left.x_{\|}=x_{1}, \ldots x_{d-1}\right)$. Their interaction with $\varphi$ shall be described here by a potential $V$, local in $x_{d}$, which, in view of the previous assumptions, will be a function of $x_{d}$ concentrated on the regions occupied by the mirrors, and vanishing elsewhere. Thus the form of $S_{I}$ shall be:

$$
\mathcal{S}_{I}(\varphi)=\frac{1}{2} \int d^{d+1} x d^{d+1} x^{\prime} \delta\left(x_{d}-x_{d}^{\prime}\right) V\left(x_{d}, x_{0}-x_{0}^{\prime}, x_{\|}-x_{\|}^{\prime}\right) \varphi(x) \varphi\left(x^{\prime}\right),
$$

which, as well as $S_{0}$, is invariant under translations in all the spacetime coordinates except $x_{d}$, namely, under the shifts $x_{\mu} \rightarrow x_{\mu}+c_{\mu}$ with $\mu=$ $0,1, \ldots, x_{d-1}, c_{\mu}=$ constant. Note that we have included a non-local dependence of the potential on the temporal and parallel coordinates, in order to describe more general responses of the mirrors.

The fact that $V$ is concentrated around each mirror, for $x_{d} \in\left[a_{1}, b_{1}\right]$ and $x_{d} \in\left[a_{2}, b_{2}\right]$, say, may be made explicit by writing it in terms of two functions, $V_{1}$ and $V_{2}$, with support on the intervals $\left[0, \delta_{1}\right]$ and $\left[0, \delta_{2}\right]$, respectively, such that:

$$
\begin{aligned}
V\left(x_{d}, x_{0}-x_{0}^{\prime}, x_{\|}-x_{\|}^{\prime}\right) & =V_{1}\left(x_{d}-a_{1}\right) \lambda_{1}\left(x_{0}-x_{0}^{\prime}, x_{\|}-x_{\|}^{\prime}\right) \\
& +V_{2}\left(x_{d}-a_{2}\right) \lambda_{2}\left(x_{0}-x_{0}^{\prime}, x_{\|}-x_{\|}^{\prime}\right) .
\end{aligned}
$$

The functions $\lambda_{i}$ characterize the response of each mirror. We regard the distances $\delta_{i}=b_{i}-a_{i}, i=1,2$ as the 'sizes' (i.e., widths) of the mirrors, while $l \equiv a_{2}-b_{1}$ is the distance between them.

To give sense to the forthcoming steps, we confine the system to a $d$ dimensional spatial box (containing the mirror) such that the field satisfies Dirichlet conditions on all the $2 d$ boundaries. That box is assumed to have 
sides of equal length, $L_{\|}$, for the $d-1$ coordinates which are parallel to the plates: $-\frac{L_{\|}}{2} \leq x_{i} \leq \frac{L_{\|}}{2}, i=1, \ldots, d-1$, while for the remaining spatial coordinate, $\left|x_{d}\right| \leq \frac{L}{2}$, with $L$ not necessarily equal to $L_{\|}$. Besides, the $x_{0}$ coordinate is also assumed to have a finite range, $x_{0} \in[-T / 2, T / 2]$, and the field to satisfy periodic boundary conditions on that interval.

We have represented, in Figure 1, our previous conventions and notations about the mirrors' configurations, from the point of view of the $x_{d}$ coordinate. Regarding the shape of the potentials, the usual, potential barrier case has been depicted in Figure 1, since that it the case when considering imperfect Dirichlet conditions. However, the derivation below is independent of that assumption, and indeed, one can even consider potential wells.

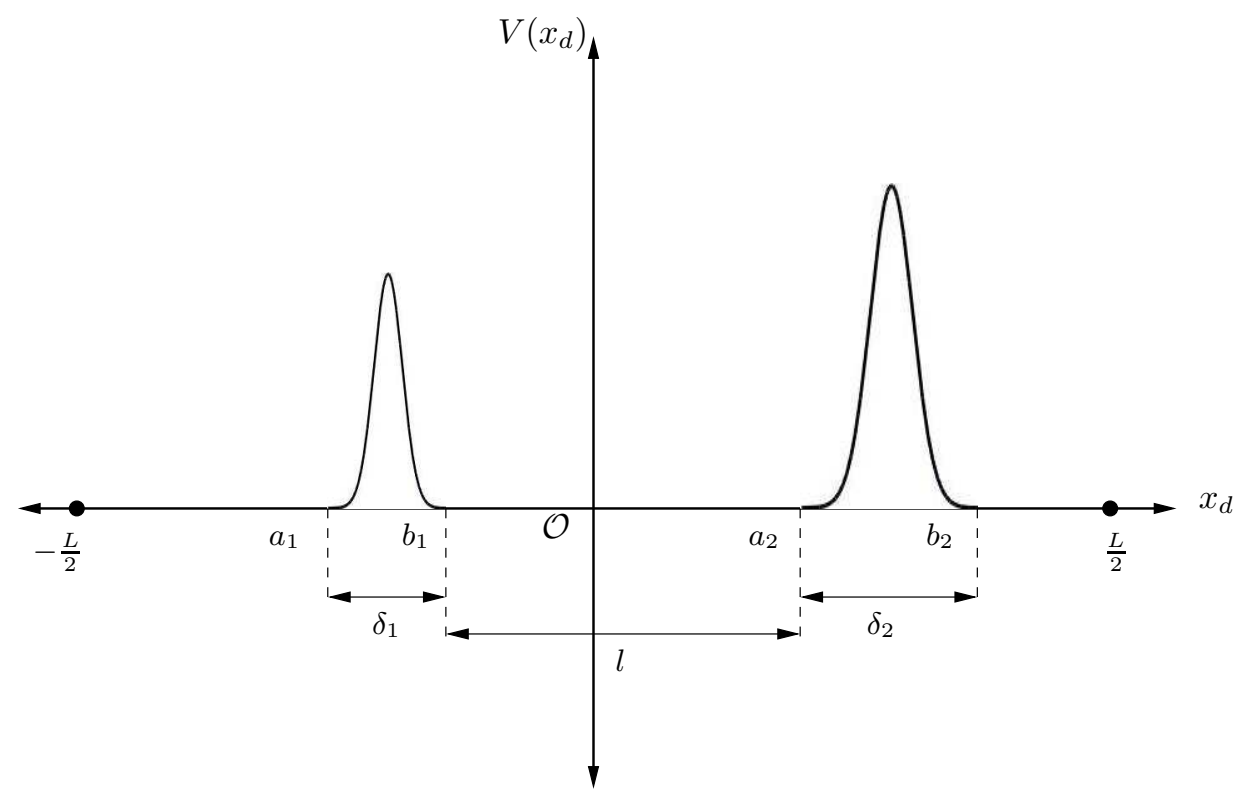

Figure 1: The potential $V\left(x_{d}\right)$, using a typical profile for the mirrors.

The vacuum energy per $(d-1)$-dimensional mirror volume, $\mathcal{E}$, relative to the vacuum energy in the absence of the mirrors $(V=0)$, for a finite $L$, can be expressed in terms of two Euclidean vacuum persistence amplitudes:

$$
\mathcal{E}=\frac{1}{2} \lim _{T, L_{\|} \rightarrow \infty}\left(\frac{1}{T L_{\|}^{d-1}} \log \frac{\mathcal{Z}}{\mathcal{Z}_{0}}\right)
$$

where $\mathcal{Z}$, the vacuum amplitude corresponding to $S$, can be written as the functional integral:

$$
\mathcal{Z}=\int \mathcal{D} \varphi e^{-S(\varphi)}
$$


while $\left.\mathcal{Z}_{0} \equiv \mathcal{Z}\right|_{V \rightarrow 0}$. Both path integrals are performed over the space of fields vanishing on the boundaries of the spatial box.

The exact -albeit formal- solution for the $\mathcal{Z}$ integral may then be given in terms of a functional determinant:

$$
\mathcal{Z}=(\operatorname{det} \mathcal{T})^{-\frac{1}{2}}
$$

with $\mathcal{T} \equiv-\partial^{2}+m^{2}+V$, an operator acting on functions of $x \in \mathbb{R}^{d+1}$ which vanish on all the boundaries of the box, and $\partial^{2} \equiv \partial_{\mu} \partial_{\mu}, \mu=0,1, \ldots, d$.

Separation of variables allows one to reduce the problem to another one for an operator acting on functions of just one variable, $x_{d}$, but dependent on a momentum vector $k=\left(k_{0}, \ldots, k_{d-1}\right)$. The determinant becomes then block-diagonal:

$$
\operatorname{det} \mathcal{T}=\prod_{k} \operatorname{det} \tilde{\mathcal{T}}(k)
$$

with $\tilde{\mathcal{T}}(k)=-\partial_{d}^{2}+\Omega^{2}(k)+\widetilde{V}\left(x_{d}, k\right), \Omega(k) \equiv \sqrt{k^{2}+m^{2}}$, which now acts on functions of $x_{d}$ which vanish at $x_{d}= \pm \frac{L}{2}$. For a potential of the form given in Eq4 we have

$$
\widetilde{V}\left(x_{d}, k\right)=V_{1}\left(x_{d}-a_{1}\right) \widetilde{\lambda}_{1}(k)+V_{2}\left(x_{d}-a_{2}\right) \widetilde{\lambda}_{2}(k) \equiv \widetilde{V}_{1}\left(x_{d}, k\right)+\widetilde{V}_{2}\left(x_{d}, k\right) .
$$

The values of $k$ become continuous and unbounded for the limit in (5), which we take now:

$$
\mathcal{E}=\frac{1}{2} \int \frac{d^{d} k}{(2 \pi)^{d}} \log \left[\frac{\operatorname{det} \widetilde{\mathcal{T}}(k)}{\operatorname{det} \widetilde{\mathcal{T}}_{0}(k)}\right]
$$

with $\left.\widetilde{\mathcal{T}}_{0} \equiv \widetilde{\mathcal{T}}\right|_{\widetilde{V}=0}$.

It is interesting, at this stage, to note that the only change one should make if one wanted to calculate the free energy per unit volume, $\Gamma$, for the same system at a finite temperature $\beta^{-1}$ would be to keep the $x_{0}$ coordinate finite, with $T=\beta$. The integral over $k_{0}$ would then become a sum over the discrete Matsubara frequencies $\omega_{n}=\frac{2 \pi n}{\beta}$. Thus

$$
\Gamma=\frac{1}{2 \beta} \sum_{n=-\infty}^{\infty} \int \frac{d^{d-1} k_{\|}}{(2 \pi)^{d-1}} \log \left[\frac{\operatorname{det} \widetilde{\mathcal{T}}\left(\omega_{n}, k_{\|}\right)}{\operatorname{det} \widetilde{\mathcal{T}}_{0}\left(\omega_{n}, k_{\|}\right)}\right],
$$

where $k_{\|}=\left(k_{1}, \ldots, k_{d-1}\right)$. Nevertheless, because the only important difference between the evaluation of the energy or the free energy emerges after the evaluation of the integrand, we just consider the energy, commenting on the analogue result for the free energy at the end. 
Note that the energy $\mathcal{E}$, besides being defined for a compact $x_{d}$ coordinate $\left(\left|x_{d}\right| \leq \frac{L}{2}\right)$, still contains a contribution from the mirrors' self energies. One possible way to get rid of those contributions is to consider the force (per unit volume) $\mathcal{F}(L)$, and its $L \rightarrow \infty$ limit, the usual, $L$-independent Casimir force, $\mathcal{F}_{C} \equiv \mathcal{F}(\infty)$ :

$$
\mathcal{F}(L)=-\frac{\partial \mathcal{E}}{\partial l}, \quad \mathcal{F}_{C} \equiv \lim _{L \rightarrow \infty} \mathcal{F}(L) .
$$

To find the ratio between determinants appearing in (15), we apply GelfandYaglom's (GY) theorem, which (after a rescaling and shift of coordinates) allows we to write it as follows:

$$
\frac{\operatorname{det} \widetilde{\mathcal{T}}(k)}{\operatorname{det} \widetilde{\mathcal{T}}_{0}(k)}=\frac{\psi\left(\frac{L}{2}\right)}{\psi_{0}\left(\frac{L}{2}\right)},
$$

where $\psi$ and $\psi_{0}$ are solutions, respectively, of the homogeneous equations:

$$
\widetilde{\mathcal{T}}(k) \psi\left(x_{d}\right)=0, \quad \widetilde{\mathcal{T}}_{0}(k) \psi_{0}\left(x_{d}\right)=0,
$$

such that $\psi\left(-\frac{L}{2}\right)=0, \psi^{\prime}\left(-\frac{L}{2}\right)=1$, and identical conditions for $\psi_{0}$.

Coming back to (12), we note that,

$$
\mathcal{F}(L)=-\frac{1}{2} \int \frac{d^{d} k}{(2 \pi)^{d}} \frac{\partial}{\partial l} \log \left[\frac{\psi\left(\frac{L}{2}\right)}{\psi_{0}\left(\frac{L}{2}\right)}\right],
$$

the Casimir force obtained afterwards by taking the $L \rightarrow \infty$ limit.

In fact, because of the logarithmic derivative above, any $l$ dependent factor may be dropped, in particular $\psi_{0}$, which is independent of $\widetilde{V}$, and hence of $l$.

Thus the next step is the calculation of $\psi\left(\frac{L}{2}\right)$ from the second order differential equation; in what follows, we shall use $x$ to denote the $x_{d}$ variable, since the problem is essentially one-dimensional. We first note some properties of the solutions to the homogeneous equation above, without making any assumption yet about $\widetilde{V}$, except that it is regular enough as to make the existence and uniqueness theorem for the second order homogeneous equation valid. Thus, given the values of $\psi$ and its derivative $\psi^{\prime}$ at a single point, $x_{i}$, say, their values at any other point, $x_{f}$, shall be uniquely determined. Introducing the two-component $x$-dependent vector $\Psi(x)=\left(\begin{array}{c}\psi(x) \\ \psi^{\prime}(x) / \Omega\end{array}\right)$, where we divided by $\Omega$ in order for the components to have equal dimensions, after some (linear) algebra we may write:

$$
\Psi\left(x_{f}\right)=A\left(x_{f}, x_{i}\right) \Psi\left(x_{i}\right)
$$


where the matrix $A$ can be written as follows:

$$
A\left(x_{f}, x_{i}\right)=U\left(x_{f}\right) U^{-1}\left(x_{i}\right)
$$

with

$$
U(x)=\left(\begin{array}{cc}
\chi_{1}(x) & \chi_{2}(x) \\
\chi_{1}^{\prime}(x) / \Omega & \chi_{2}^{\prime}(x) / \Omega
\end{array}\right) .
$$

Here, $\chi_{1}$ and $\chi_{2}$ denote two independent solutions of the homogeneous equation, forming a basis of solutions (it can be shown that $A$ is independent of the choice of basis). From the fact that these two functions are independent solutions of the homogeneous equation, it follows that $\operatorname{det} U(x)$, their (scaled) Wronskian determinant, is a non vanishing constant. Therefore, we obtain the property:

$$
\operatorname{det} A\left(x_{f}, x_{i}\right)=1 \text {. }
$$

Note that $\psi\left(\frac{L}{2}\right)$ may be written in terms of a single matrix elements of $A$, as follows:

$$
\psi\left(\frac{L}{2}\right)=A_{12}(L / 2,-L / 2),
$$

where we dropped $l$ independent factors.

For the particular case we are considering, namely, a potential which vanishes everywhere except at the region occupied by the mirrors, the previous expression may be rendered in a more explicit fashion. To that order, it is convenient to make repeated use of (16) to write $A$ as a product of matrices corresponding to subintervals. Those factors are completely determined by the potential inside the respective intervals; thus, it is either just an $A_{0}$ factor, where the potential vanishes, or it is determined by the solution of a homogeneous equation with $\widetilde{V}$ replaced by either $\widetilde{V}_{1}$ or $\widetilde{V}_{2}$. Thus:

$$
\begin{gathered}
A(L / 2,-L / 2)=A^{(0)}\left(L / 2, b_{2}\right) A^{\left(V_{2}\right)}\left(b_{2}, a_{2}\right) \\
\times A^{(0)}\left(a_{2}, b_{1}\right) A^{\left(V_{1}\right)}\left(b_{1}, a_{1}\right) A^{(0)}\left(a_{1},-L / 2\right)
\end{gathered}
$$

where we have indicated which potential each factor corresponds to. The matrix $A^{(0)}\left(x_{f}, x_{i}\right)$, corresponding to a null potential on the interval $\left[x_{i}, x_{f}\right]$ can be immediately found, by using, for example, the independent functions $\chi_{1}(x)=e^{\Omega x}$ and $\chi_{2}(x)=e^{-\Omega x}$, the result being:

$$
A^{(0)}\left(x_{f}, x_{i}\right) \equiv A^{(0)}(\Delta x)=\left(\begin{array}{cc}
\cosh (\Omega \Delta x) & \sinh (\Omega \Delta x) \\
\sinh (\Omega \Delta x) & \cosh (\Omega \Delta x)
\end{array}\right),
$$

$\Delta x \equiv x_{f}-x_{i}$. Besides, note that the only nontrivial factors are $A^{\left(V_{1,2}\right)} \equiv$ $A^{(1,2)}, 2 \times 2$ matrices depending on each potential, the wave vector $k$; they depend on $a_{i}, b_{i}$ only through their differences, $\delta_{i}$. 
Taking into account the results above, we may write a quite compact expression for $\psi(L / 2)$ :

$$
\psi(L / 2)=u_{2}^{T} A^{(2)} A^{(0)}(l) A^{(1)} v_{1},
$$

where we introduced the 2-component vectors, $u_{i} v_{i}$,

$$
u_{i}=\left(\begin{array}{c}
\cosh \left(\Omega l_{i}\right) \\
\sinh \left(\Omega l_{i}\right)
\end{array}\right), \quad v_{i}=\left(\begin{array}{c}
\sinh \left(\Omega l_{i}\right) \\
\cosh \left(\Omega l_{i}\right)
\end{array}\right)
$$

and $l_{1}, l_{2}$ are the distances from the mirrors to the boundaries of the spatial box. We shall assume now that $b_{1}=-l / 2$ and $a_{2}=l / 2$, so that the internal faces of the mirrors are symmetrically disposed with respect to the origin (as well as the boundaries at $\pm L / 2)$. Then $l_{1,2}=(L-l) / 2-\delta_{1,2}$. Note that, in (23)), the only factors that depend on $l$ are $u_{2}, v_{1}$, and $A^{(0)}$.

To calculate the logarithmic derivative of $\psi(L / 2)$, we first note that

$$
\begin{aligned}
\frac{\partial \psi(L / 2)}{\partial l} & =\Omega\left[u_{2}^{T} A^{(2)} B^{(0)} A^{(1)} v_{1}\right. \\
& \left.-\frac{1}{2}\left(v_{2}^{T} A^{(2)} A^{(0)} A^{(1)} v_{1}+u_{2}^{T} A^{(2)} A^{(0)} A^{(1)} u_{1}\right)\right]
\end{aligned}
$$

where

$$
B^{(0)} \equiv\left(\begin{array}{cc}
\sinh (\Omega l) & \cosh (\Omega l) \\
\cosh (\Omega l) & \sinh (\Omega l)
\end{array}\right)
$$

Rather than writing the rather lengthy expression for the logarithmic derivative for a finte $L$, we directly present its $\frac{L}{l} \rightarrow \infty$ limit. Introducing the (constant) vector $w_{1}=\frac{1}{\sqrt{2}}\left(\begin{array}{l}1 \\ 1\end{array}\right)$, and matrix $C=\left(\begin{array}{cc}-1 & 1 \\ 1 & -1\end{array}\right)$ :

$$
\left[\frac{\partial \log \psi(L / 2)}{\partial l}\right]_{L \rightarrow \infty}=\Omega e^{-\Omega l} \frac{w_{1}^{T} A^{(2)} C A^{(1)} w_{1}}{w_{1}^{T} A^{(2)} A^{(0)} A^{(1)} w_{1}} .
$$

It becomes clear from the last expression that it is convenient to perform a change of basis in all the matrices involved. Indeed, rotating to the basis $w_{1}$, $w_{2}$, with $w_{2}=\frac{1}{\sqrt{2}}\left(\begin{array}{c}1 \\ -1\end{array}\right)$, and denoting by $T^{(0,1,2)}$ and $D$ the form adopted by $A^{(0,1,2)}$ and $C$ in the new basis, respectively, we see that:

$$
\left[\frac{\partial \log \psi(L / 2)}{\partial l}\right]_{L \rightarrow \infty}=\Omega e^{-\Omega l} \frac{w_{1}^{T} T^{(2)} D T^{(1)} w_{1}}{w_{1}^{T} T^{(2)} T^{(0)} T^{(1)} w_{1}} .
$$


More explicitly,

$$
\begin{aligned}
{\left[\frac{\partial \log \psi(L / 2)}{\partial l}\right]_{\frac{L}{l} \rightarrow \infty} } & =\frac{-2 \Omega e^{-2 \Omega l} \frac{T_{12}^{(2)}}{T_{11}^{(2)}} \frac{T_{21}^{(1)}}{T_{11}^{(1)}}}{1+\frac{T_{12}^{(2)}}{T_{11}^{(2)}} \frac{T_{21}^{(1)}}{T_{11}^{(1)}} e^{-2 \Omega l}} \\
& =\frac{\partial}{\partial l} \log \left[1+\frac{T_{12}^{(2)}}{T_{11}^{(2)}} \frac{T_{21}^{(1)}}{T_{11}^{(1)}} e^{-2 \Omega l}\right] .
\end{aligned}
$$

Finally, we arrive to an expression for the force per unite volume,

$$
\mathcal{F}=-\frac{1}{2} \int \frac{d^{d} k}{(2 \pi)^{d}} \frac{\partial}{\partial l} \log \left[1+\frac{T_{12}^{(2)}}{T_{11}^{(2)}} \frac{T_{21}^{(1)}}{T_{11}^{(1)}} e^{-2 \Omega l}\right],
$$

which is the main result of this paper.

It is worth interpreting, at this point, the meaning of the ratios between matrix elements of $T^{(1,2)}$ appearing in (30). From the original definition of $A$, and implementing the change of basis, one sees that the matrix elements of $T^{(i)}, i=1,2$ relate $\psi$ and $\psi^{\prime}$ just to the right of the mirror with the function and its derivative just to the left, but in a mixed fashion. Indeed, in the new basis, it relates functions such that $\psi^{\prime}=\Omega \psi$ or $\psi^{\prime}=-\Omega \psi$ from one face of the mirror to the other. Thus, the matrix elements of $T^{(i)}$ connect solutions of the form $e^{ \pm \Omega x}$ on both sides of the mirror.

We can make contact with the usual expression of Lifshitz formula from the following observation: Identifying the Euclidean function $e^{\Omega x}$ with the (Euclidean version of) a left moving mode, and $e^{-\Omega x}$ with a right moving mode, we see that $r_{R}^{(1)}=\frac{T_{21}^{(1)}}{T_{11}^{(1)}}$ is the (Euclidean) reflection coefficient associated to the left mirror, for incoming right modes. In the same fashion, $r_{L}^{(2)}=-\frac{T_{12}^{(2)}}{T_{11}^{(2)}}$ corresponds to the reflection coefficient of the right mirror, for incoming right modes (for non-symmetric potentials one may have $r_{R}^{(i)} \neq r_{L}^{(i)}$ ). In terms of these coefficients the Casimir force reads

$$
\mathcal{F}=-\frac{1}{2} \int \frac{d^{d} k}{(2 \pi)^{d}} \frac{\partial}{\partial l} \log \left[1-r_{R}^{(1)} r_{L}^{(2)} e^{-2 \Omega l}\right],
$$

which is of the same form that the Lifshitz formula for a scalar field. As already mentioned in Section 2, the case of finite temperature is obtained by replacing the integral over $k_{0}$ by a sum over dicrete Matsubara frequencies.

\section{$3 \quad$ Examples}

The matrices $T^{(i)}$ appearing in the final expression of the force can be explicitly computed in several particular cases. For instance, let us assume that a 
mirror is described by a square potential barrier of height $\widetilde{V}_{0}(k)$ and width $\delta$ [13. A straightforward calculation yields:

$$
\begin{aligned}
& T_{11}=\cosh (\bar{\Omega} \delta)+\frac{\Omega^{2}+\bar{\Omega}^{2}}{2 \Omega \bar{\Omega}} \sinh (\bar{\Omega} \delta) \\
& T_{22}=\cosh (\bar{\Omega} \delta)-\frac{\Omega^{2}+\bar{\Omega}^{2}}{2 \Omega \bar{\Omega}} \sinh (\bar{\Omega} \delta) \\
& T_{12}=-T_{21}=\frac{\bar{\Omega}^{2}-\Omega^{2}}{2 \Omega \bar{\Omega}} \sinh (\bar{\Omega} \delta),
\end{aligned}
$$

where $\bar{\Omega}^{2}=\Omega^{2}+\widetilde{V}_{0}(k)$. Note that for these symmetric potentials, the left and right reflection coefficients coincide, that is $r_{L}^{(i)}=r_{R}^{(i)}$.

The Casimir force between two mirrors described by such potentials is obtained by replacing the corresponding matrix elements into Eq.(30), and the final result is in agreement with Ref.[13]. We show explicitly the expression of the force only in some limiting cases: for very thick slabs with $\delta_{i}>>l$, the vacuum force reads

$$
\mathcal{F}=-\frac{1}{2} \int \frac{d^{d} k}{(2 \pi)^{d}} \frac{\partial}{\partial l} \log \left[1-\frac{\widetilde{V}_{0}^{(1)} \widetilde{V}_{0}^{(2)}}{\left(\Omega+\bar{\Omega}^{(1)}\right)^{2}\left(\Omega+\bar{\Omega}^{(2)}\right)^{2}} e^{-2 \Omega l}\right] .
$$

On the other hand, one can also obtain from the general expression (32) the $T$ matrix associated to a singular potential of the form $\widetilde{V}(x, k)=\widetilde{\lambda}(k) \delta(x)$, where $\delta(x)$ is the Dirac $\delta$-function. Indeed, taking the limits $\widetilde{V}_{0}(k) \rightarrow \infty$ and $\delta \rightarrow 0$ with $\widetilde{V}_{0}(k) \delta \rightarrow \widetilde{\lambda}(k)$, the $T$ matrix becomes:

$$
T=\left(\begin{array}{cc}
1+\frac{\widetilde{\lambda}}{2 \Omega} & \frac{\tilde{\lambda}}{2 \Omega} \\
-\frac{\widetilde{\lambda}}{2 \Omega} & 1-\frac{\widetilde{\lambda}}{2 \Omega}
\end{array}\right)
$$

thus, the force between two zero-width mirrors, with functions $\widetilde{\lambda}_{1}(k)$ and $\widetilde{\lambda}_{2}(k)$, at a distance $l$ appart is

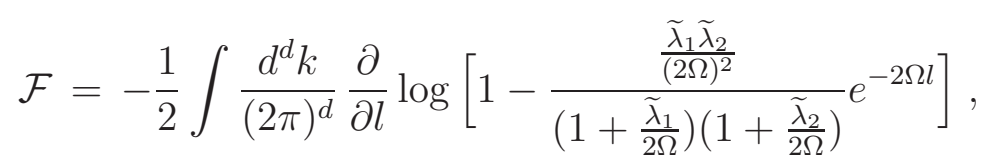

which reproduces a well known result [10, 13, 14].

\section{Conclusions}

In this paper we presented a simple derivation of Lifshitz formula, based on the use of GY theorem to compute the functional determinant of an operator 
of the form $-\partial^{2}+m^{2}+V$, where $V$ is a background potential that models the imperfect mirrors.

The fact that the Casimir force between flat mirrors can be written just in terms of the reflection coefficients of each mirror, is quite transparent in this derivation. Indeed, after separation of variables the problem reduces to the calculation of the functional determinant of the one dimensional operator $\widetilde{\mathcal{T}}(k)=-\partial_{d}^{2}+\Omega^{2}(k)+\widetilde{V}\left(x_{d}, k\right)$. GY formula implies that this determinant is proportional to $\psi(L / 2)$, where $\psi\left(x_{d}\right)$ is a solution of $\widetilde{\mathcal{T}}(k) \psi\left(x_{d}\right)=0$, satisfying the initial conditions $\psi(-L / 2)=0, \psi^{\prime}(-L / 2)=1$. The key observation is that one can evaluate $\psi$ and its derivative on the right side of each mirror in terms of their values on the left side, by means of a transfer matrix determined by the potential describing the mirror. This matrix contains all the information of the potential upon which the functional determinant may depend. On top of that, the Casimir force only depends on some particular ratio of elements of the transfer matrix. Hence, the apparent dependence on the function that describes the mirror collapses to a single number (which may of course depend on frequency or momentum).

We wish to point out that the self-energies of the mirrors do not appear in the expression for the force, since the derivative with respect to the distance between mirrors erases them out. The same would happen if we considered the difference between the energies corresponding to two different distances.

Finally, we note that expression (30) for the force can be thought of as a generalization of Lifshitz formula to the case of mirrors described by potentials of the form $V=V\left(x_{d}, x_{0}-x_{0}^{\prime}, x_{\|}-x_{\|}^{\prime}\right)$, that may include not only potential barriers but also potential wells. In this case, equation (30) holds true, even when its interpretation in terms of reflection coefficients in the real time formulation would be problematic, because of the possible existence of bound states. Moreover, potentials of this form are generated in a wide class of theoretical models in which the vacuum field is coupled to the internal degrees of freedom of the mirrors, and therefore this approach provides an explicit link between the force and the microscopic interactions.

\section{Acknowledgements}

This work was supported by ANPCyT, CONICET, UBA and UNCuyo. 


\section{References}

[1] H. B. G. Casimir, Indag. Math. 10, 261 (1948) [Kon. Ned. Akad. Wetensch. Proc. 51, 793 (1948)] [Front. Phys. 65, 342 (1987)] [Kon. Ned. Akad. Wetensch. Proc. 100N3-4, 61 (1997)].

[2] E. M. Lifshitz, Sov. Phys. JETP 2, 73 (1956).

[3] P. W. Milonni, The Quantum Vacuum, Academic Press, San Diego, 1994; M. Bordag, U. Mohideen, and V. M. Mostepanenko, Phys. Rep. 353, 1 (2001); K. A. Milton, The Casimir Effect: Physical Manifestations of the Zero-Point Energy (World Scientific, Singapore, 2001); S. Reynaud et al., C. R. Acad. Sci. Paris IV-2, 1287 (2001); K. A. Milton, J. Phys. A: Math. Gen. 37, R209 (2004); S.K. Lamoreaux, Rep. Prog. Phys. 68, 201 (2005); M. Bordag, G.L. Klimchitskaya, U. Mohideen, and V. M. Mostepanenko, Advances in the Casimir Effect, Oxford University Press, Oxford, 2009.

[4] T.G. Philbin, New J. Phys. 12, 123008 (2010).

[5] See Bordag et al (2001), in Ref. [3].

[6] C. Genet, A. Lambrecht, and S. Reynaud, Phys. Rev. A67, 043811 (2003).

[7] D. Kupiszewska, Phys. Rev. A46, 2286 (1992); F.C. Lombardo, A. Rubio Lopez, and F.D. Mazzitelli, in preparation.

[8] N. Graham, R. L. Jaffe, V. Khemani, M. Quandt, O. Schroeder, and H. Weigel, Nucl. Phys. B 677, 379 (2004).

[9] C. D. Fosco, F. C. Lombardo, and F. D. Mazzitelli, Phys. Rev. D 77, 085018 (2008).

[10] C. D. Fosco, F. C. Lombardo, and F. D. Mazzitelli, Phys. Lett. B 669, $371(2008)$

[11] I. M. Gelfand and A. M. Yaglom, J. Math. Phys. 1, 48 (1960).

[12] G. V. Dunne, J. Phys. A 41, 304006 (2008).

[13] These piecewise constant potentials with $k$-independent $\widetilde{V}_{0}$ have been considered in I. V. Fialkovsky, V. N. Markov, and Y. M. Pis'mak, J. Phys. A: Math. Theor. 43, 365401 (2010).

[14] K. A. Milton, J. Phys. A 37, 6391 (2004) 